\title{
Wirtschaftliche Untergangsszenarien und neoliberale Reformen
}

\section{Großideologien und das lästige Problem politischer Durchsetzung}

Die gegenwärtige Wiederkehr offen zur Schau getragener protektionistischer Politik gilt vielen Beobachtern der USA als Symptom einer Epochenschwelle. ${ }^{1}$ Seit dem Niedergang der New Deal Era, so etwa eine beliebte historische Einordnung gegenwärtiger amerikanischer Handelspolitik, war die wesentliche Konfliktlinie eine der Anerkennung, nicht der Verteilung. Für die Zeit zwischen den späten 1970er Jahren und der Wirtschafts- und Finanzkrise nach 2008 gilt neoliberale Verteilungspolitik als politischer Grundkonsens zwischen rechten und linken Regierungen: »[B]eide Blöcke unterstützten 'freien Handel«, niedrige Unternehmenssteuern, beschnittene Arbeitnehmerrechte, das Primat von Shareholder-Interessen, Winner-takes-all-Vergütung und die Deregulierung von Finanzmärkten. Beide Blöcke wählten Führungsriegen, die sgrand bargains ‘ anstrebten, um Wohlfahrtsleistungen zu kürzen. « ${ }^{2}$ Dieses relativ kohärente Bündel politisch-ökonomischer Reformen, das in der vergleichenden Forschung häufig als eine Art Minimalaufstellung neoliberaler Politik gilt, konnte politische Gräben überspannen, weil es zum neuen Grundverständnis guter Wirtschaftspolitik wurde. ${ }^{3}$ Bestimmende Konflikte entsponnen sich hingegen um "gesellschaftliche " Probleme, etwa um die Geschlechtergerechtigkeit, Familienstrukturen und die Migration.

Ein Stück weit sind derart griffige Retrospektiven auf die Wirtschaftspolitik der letzten 50 Jahre eine Folge der Wirtschafts- und Finanzkrise. ${ }^{4}$ Dieser »Kulminationspunkt ", so scheint es, hat die Debatte über die grundlegende Richtung der Wirtschaftspolitik der letzten Jahrzehnte verebben lassen. ${ }^{5}$ Hochstilisierte Darstellungen sind ein Grund dafür, dass die gegenwärtige US-Politik als Bruch mit der Vergangenheit verstanden wird. Mein Beitrag ist kein Versuch, Antworten auf strukturelle Fragen eine widersprüchlichere Detailgeschichte vorzuhalten. ${ }^{6}$ Vielmehr argumentiere ich erstens, dass die Charakterisierung gegenwärtiger politischer Entwicklun-

1 Für hilfreiche Kommentare danke ich den Teilnehmerinnen und Teilnehmern des MPIfGInstitutsseminars im Sommer 2018 und der Fruhjahrstagung der DGS-Sektion Wirtschaftssoziologie 2018 in Graz. Der Artikel entstand während eines Gastaufenthalts am Department of Sociology der Columbia University, wofür ich Josh Whitford danke.

2 Fraser 2017; Übersetzung T.E.

3 Fourcade, Babb 2002; Prasad 2006.

4 Rodgers 2018.

5 Eine Ausarbeitung der These vom Kulminationspunkt 2007/2008 findet sich bei Streeck 2011.

6 Siehe zu diesem Fehlschluss klassisch: Whitehead 1967 [1926], S. 50-51. 
gen mithilfe eines stilisierten Modells der Vergangenheit in die Irre führt. Abseits durchaus pfadbrechender handelspolitischer Rhetorik wiederholt die Trump-Administration praktisch ein seit den 1980er Jahren geläufiges Muster in der Wirtschaftspolitik - wirtschaftsliberale Politik nach innen und selektiv interventionistische Politik nach außen. Am Beispiel des Economic Recovery Tax Acts (ERTA) von 1981, der Schlüsselreform in Ronald Reagans Wirtschaftspolitik, zeigt mein Beitrag, wie die Durchsetzung neoliberaler Reformen mithilfe wirtschaftlicher Niedergangserzählungen Regierungen unter handelspolitischen Handlungsdruck setzen kann. Zweitens ergänzt mein Beitrag die Analyse politischer Reformprozesse um ein konzeptuelles Argument. Geschichten über die Zukunft haben einen - verglichen mit materiellen Interessen und institutionellen Strukturen - selten diskutierten Einfluss auf politische Prozesse. ${ }^{7}$ Die hier vorgelegte politikfeldübergreifende Betrachtung der Wirkung von Niedergangserzählungen in der Steuer- und Handelspolitik zeigt, dass Geschichten über die Zukunft Koalitionen verschieben und ein Eigenleben entwickeln können. Methodisch stützt sich mein Artikel auf die Analyse von Archivmaterial der Reagan-Präsidentschaft und des amerikanischen Kongresses sowie von zeitgenössischen Presseberichten zwischen den späten 1970er und den späten 1980er Jahren.

Mit sozialwissenschaftlichen Ansätzen, die den Einfluss von Ideen auf die Politik betonen, argumentiere ich, dass ein Wandel ideeller Konstrukte einen Wandel im politischen Handeln bedingen kann. ${ }^{8}$ Gegen sie argumentiere ich, dass Reformer die Großideologien, an denen sie sich orientieren, situationsbedingt anreichern und gelegentlich pragmatisch mit den Ideen ihrer politischen Gegner verbinden. Während es nicht einmal falsch sein mag, dass neoliberale Ideen seit den späten 1970er Jahren einen hegemonialen Einfluss auf die Wirtschaftspolitik reicher westlicher Länder hatten, ${ }^{9}$ hilft die Analyse von Großideologien nur sehr bedingt weiter, wenn die aus ihnen erwachsenen Debatten und Policies Flickwerk sind. In der neueren Forschung zu neoliberalen Reformen hat dies einige Beobachter so weit gebracht, dass sie eine separate Untersuchung neoliberaler Ideen und neoliberaler Politik fordern. ${ }^{10}$ Konstruktiver haben Popp Berman und Pagnucco in ihren Untersuchungen von Steuerdebatten im amerikanischen Kongress vorgeschlagen, unterschiedliche Analysestrategien für unterschiedliche Phasen des politischen Prozesses zu verwenden. ${ }^{11}$ Während die Untersuchung von Großideologien und Paradigmen in der frühen Formulierungsgeschichte von Gesetzen aufschlussreich sei, bedürfe es in späteren Phasen politischer Durchsetzung Analysen pragmatischer Koalitionsbildung und diskursiver Rechtfertigung. Genereller wird in der historisch-institutionalistischen Sozialwissenschaft seit kurzem häufiger gefordert, distinkte theoretische

7 Beckert 2019.

8 Siehe zusammenfassend zur neueren politisch-soziologischen Literatur zum Einfluss von Ideen auf politische Prozesse: Hall 1993; Blyth 2002; Schmidt 2008.

9 Vgl. hierzu etwa Fraser 2017.

10 Rodgers 2018.

11 Popp Berman, Pagnucco 2010.

Leviathan, 47. Jg., 2/2019 
Ansätze für die Analyse der »unordentlicheren « Phasen der Durchsetzung und Implementation von Gesetzen zu entwickeln. ${ }^{12}$

Für die Untersuchung wirtschaftsliberaler Reformen macht eine solche stufenweise Analysestrategie viel Sinn, da sie regelmäßig an die politischen Grenzen »sauberer « Umsetzung stoßen. Policies zur wirtschaftlichen » Modernisierung « bedeuten in der Regel erhebliche kurzfristige Verluste für signifikante Teile der Bevölkerung sowie für politisch einflussreiche Gruppen. ${ }^{13}$ Ist die Zustimmung der wahrscheinlichen Verlierer von Reformen zur Umsetzung nötig, weichen Reformer notgedrungen von ihren Großideologien ab, um Koalitionen zu ihren Gunsten zu verschieben. Die wenigsten Textilarbeiter sorgen sich um die Logik komparativer Vorteile und die wenigsten Stahlmanager um die Segnungen von knowledge-based growth. ${ }^{14}$ Ein Rechtfertigungsnarrativ zur Durchsetzung wirtschaftlicher Reformen, von dem seit den späten 1970er Jahren immer wieder Gebrauch gemacht wurde, bedient sich industrieller Niedergangsszenarien und der Beschwörung der Gefahren internationaler Konkurrenz. Ohne kurzfristige kollektive Entbehrungen, so die argumentative Grundstruktur, verlieren Nationen ihre industriellen und technologischen Vorteile an agilere, fleißigere oder besser organisierte Handelspartner. ${ }^{15}$ Auf genau dieses Rechtfertigungsmuster berief sich die erste Regierung Ronald Reagans zur Durchsetzung verhältnismäßig radikaler Unternehmens- und Einkommensteuersenkungen, die zur Revitalisierung einer international in Bedrängnis geratenen heimischen Industrie beitragen sollten. Genau diese Erzählungen waren es aber auch, die die US-amerikanischen Regierungen der 1980er Jahre unter Zugzwang setzten, die versprochene industrielle Revitalisierung mit einer interventionistischen Handelspolitik herbeizuführen.

Offen nach den Wirkungen einer bestimmten Ursache zu fragen statt nach den Ursachen einer bestimmten Wirkung, hat Vor und Nachteile. ${ }^{16}$ Einerseits erlaubt die politikfeldübergreifende Untersuchung die Rekonstruktion breiterer Entwicklungen. Andererseits müssen Abstriche bei der Einordnung in politikfeldspezifische

12 Siehe exemplarisch: Hacker et al. 2015; Patashnik 2008; Patashnik, Zelizer 2013.

13 Streeck, Elsässer 2016.

14 Was nicht zwingend bedeutet, dass derartige Interessengruppen deswegen »kurzsichtiger « als andere gesellschaftliche Gruppen sind. In der Literatur sowie im Diskurs zu wirtschaftlichen Reformen werden aufgeklärte, langfristig "rationale « Reformer gerne kurzfristig materiell interessierten Reformgegnern gegenübergestellt. Häufig, so meine starke Vermutung, werden dabei die Antizipationsfähigkeiten und nichtmateriellen Motive ersterer systematisch überschätzt, während die der letzteren systematisch unterschätzt werden.

15 Eine einsichtsreiche Polemik zur Beschwörung der wirtschaftlichen Konkurrenz zwischen ganzen Gesellschaften ist Paul Krugmans Aufsatz »Competitiveness: a dangerous obsession « (Krugman 1994). In der Politischen Ökonomie existiert eine Literatur zum sogenannten Competition State, einer Neudefinition der Rolle des Staates seit den 1970er Jahren, die historisch den Wohlfahrtstaat abgelöst habe. Für einen Überblick und Kritik siehe Genschel, Seelkopf 2015.

16 Gerring nennt derartige Forschungsansätze »x-centered « und diskutiert ihre Stärken und Schwächen (Gerring 2001, S. 137). 
Debatten in Kauf genommen werden. Im nächsten Abschnitt stelle ich meine Perspektive in Auseinandersetzung mit dem gegenwärtigen Forschungsstand zur Durchsetzung von Reagans erster großer Steuerreform dar. Daran anschließend zeige ich in Abschnitt 3, wie wirtschaftliche Niedergangserzählungen Einfluss in der Steuergesetzgebung gewannen und wie sie genutzt wurden, um Unterstützung für Steuerreformen zu mobilisieren. Diese Erzählungen wiederum setzten die Regierung Reagans unter Druck, protektionistische Maßnahmen einzuführen (Abschnitt 4). Dieses Wechselspiel zwischen unternehmensfreundlicher Steuer- und selektiv interventionistischer Handelspolitik im Dienste einer Revitalisierung der amerikanischen Industrie hat starke Parallelen zur gegenwärtigen amerikanischen Wirtschaftspolitik. In Abschnitt 5 werden diese Parallelen skizziert und konzeptuelle Folgerungen zur Rolle von Niedergangserzählungen in der Wirtschaftspolitik angedeutet.

\section{Das Problem der Durchsetzung neoliberaler Steuerreformen}

Im Vergleich zu anderen Politikfeldern ist die Frage nach den definierenden Wesenszügen »neoliberaler « Steuerreformen nicht einfach zu beantworten. Während etwa der Niedergang keynesianischer Globalsteuerung oder das Ende des Ausbaus wohlfahrtstaatlicher Programme seit den 1970er Jahren als relativ kohärente paradigmatische Wandlungen beschrieben werden können, ist der Wandel der Steuersysteme reicher Länder seit den 1980er Jahren von Widersprüchen durchzogen. ${ }^{17}$ Als erste Annäherung bietet es sich an, »regressive « Steuerreformen als neoliberal einzuordnen - Reformen, die auf welche Weise auch immer dafür verantwortlich sind, dass das jeweilige Steuersystem weniger von oben nach unten umverteilend wirkt. Auf den ersten Blick werden mit einer solchen Bestimmung wichtige steuerpolitische Trends der letzten drei Jahrzehnte auf einen Nenner gebracht - etwa die kontinuierliche Reduzierung von Unternehmens- und Kapitalertragssteuersätzen, die Verbreitung und Erhöhung von Verbrauchs- und Mehrwertsteuern oder die Reduzierung der Spitzensteuersätze auf Einkommen und Vermögen. ${ }^{18}$ Auf den zweiten Blick ist das Bild komplizierter. Komparativ-historisch zeichneten sich gerade stark umverteilende Volkswirtschaften durch stark regressive Steuersysteme aus. ${ }^{19}$ Und während gerade die Unternehmensbesteuerung seit 1984 OECD-weit drastisch nominal gesenkt wurde, wurden im Gegenzug regelmäßig Abschreibungsmöglichkeiten eingedämmt, sodass die Reduktion der effektiven Steuerbelastung von Unternehmen weit weniger radikal war. ${ }^{20}$

Besser lässt sich der gemeinsame Nenner von neoliberalen Steuerreformen mit einem Blick auf ihre Begründungen und Ziele bestimmen. Fairness- und Umverteilungserwägungen sowie staatliche Lenkungsambitionen haben seit den 1980er Jah-

17 Einflussreiche Arbeiten, die den paradigmatischen Wandel in der Wirtschaftspolitik seit den 1970er Jahren auf den Punkt zu bringen versuchen, sind Blyth 2002; Rodgers 2011.

18 Siehe unter vielen: Piketty, Saez 2006.

19 Lindert 2004, Kapitel 10-12; Steinmo 1989.

20 Ganghof 2000; Ganghof 2004; Swank, Steinmo 2002.

Leviathan, 47. Jg., 2/2019 
ren stückweise ihre Bedeutung als zentrale Motivationen für Änderungen in der Einkommens-, Unternehmens- und Kapitalbesteuerung verloren. ${ }^{21}$ Stattdessen wurden steuerpolitische Debatten zunehmend von ökonomischen Kriterien und Prinzipien geprägt. ${ }^{22}$

Die mit Abstand umfangreichste Literatur zur Erklärung dieses Wandels basiert auf diversen Spielarten von Diffusionsmodellen. ${ }^{23}$ Politisch, medial und akademisch enorm einflussreich sind Modelle, die die Ursache neoliberaler Steuerpolitik im globalen Wettbewerb zwischen Nationen um flüchtiges Kapital und Investitionen sehen. Je intensiver diese Konkurrenz, so die Grundidee, desto stärker verschieben Staaten ihre Finanzierung auf weniger mobile Faktoren wie Arbeit und Konsum. ${ }^{24}$ Regressive Steuerreformen wären damit schlicht rationale Reaktionen von Staaten auf gewandelte strukturelle Rahmenbedingungen. Analoge Diffusionsmodelle sind auch für ideellen Wandel formuliert worden. Duane Swank hat argumentiert, dass neoliberale Reformen seit den 1980er Jahren zu einem neuen Paradigma »moderner « Steuerpolitik wurden, das sich insbesondere durch die Signalwirkung der USamerikanischen Reform von 1986 international verbreitete. ${ }^{25}$

So wichtig die Frage nach der internationalen Diffusion neoliberaler Steuerpolitik ist, so wenig helfen Diffusionsmodelle zu verstehen, woher der politische Impuls zu diesem paradigmatischen Wandel in der Steuerpolitik kam. Insbesondere in Großbritannien und den USA, die in Diffusionsmodellen zumeist als Vorreiter gelten, wurde in den 1980er Jahren mit jahrzehntelang verfestigten Praktiken in der Steuerpolitik gebrochen. Wenn die Steuerpolitik der Nachkriegsjahrzehnte von geteilten politischen Ideen, Interessengruppen und Koalitionsstrukturen getragen wurde, dann stellt sich die Frage, wie Regierungen nach 1980 den politischen Handlungsspielraum schaffen konnten, der ihnen die Durchsetzung pfadbrechender Reformen erlaubte.

In der historischen Literatur lassen sich grob drei Erklärungen für die politische Offenheit der Steuerpolitik seit 1980 unterscheiden - elitenbasierte, wahlstrategische und industriepolitische. Sie alle setzen historisch mit der politischen Auseinandersetzung um die erste große Steuerreform der Reagan-Regierung an, dem Economic Recovery Tax Act (ERTA) von 1981. Zur Einordnung dieser Erklärungen ist ein wenig Hintergrundwissen zu seiner Struktur hilfreich.

Gemessen am statisch berechneten Einnahmeausfall, war der ERTA die größte Steuersenkung der neueren amerikanischen Geschichte, und er wird häufig als Ursprung einer Reihe regressiver Steuerreformen in den USA behandelt. ${ }^{26}$ Nur ein Jahr nach seiner Verabschiedung im August 1981 begann die Administration mit

21 Swank 2006.

22 Siehe dazu sehr einsichtsreich: Rademacher 2017.

23 Für einen Überblick: Simmons et al. 2007.

24 Siehe zu Einblicken in die weit verzweigte Literatur: Ganghof 2000; Genschel 2002; Genschel, Schwarz 2011.

25 Swank 2006.

26 Piketty 2014, S. 495-496. 
einer Serie von fünf zusätzlichen Gesetzen bis 1987, die den Haushaltslöchern entgegenwirken sollten, die der ERTA geschaffen hatte (siehe Tabelle 1). Zeitgenössische Schätzungen gingen davon aus, dass die Einnahmeausfälle durch den ERTA bis 1985 jährlich fast 2,9 Prozent des Bruttoinlandsprodukts ausgemacht hätten, was einer Reduzierung der bundesstaatlichen Steuereinnahmen von 13,3 Prozent entsprochen hätte. Im Grunde bestand das Gesetzespaket, das 1981 im ERTA mündete, seit Ronald Reagans Wahlkampagne aus drei wesentlichen Komponenten: einer Senkung des Einkommensteuersatzes, einem Anreizbündel für Unternehmensinvestitionen und Indexierungsvorkehrungen, die Steuerzahler vor hohen Inflationsraten schützen sollten. In seiner finalen Form bestand der ERTA im Kern aus einem 23-prozentigen Schnitt aller Einkommensteuersätze ab Mitte des Jahrzehnts, Indexierungsvorkehrungen und einem Bündel aus veränderten Abschreibungsregeln: dem Accelerated Cost Recovery System (ACRS), einem Investment Tax Credit (ITC) und Abschreibungsmöglichkeiten für Forschungs- und Entwicklungsaufwendungen (siehe Tabelle 2).

\section{Tabelle 1: Statische Einnahmeeffekte größerer amerikanischer Steuerreformen}

\begin{tabular}{|c|c|c|c|c|}
\hline \multirow[t]{3}{*}{ Gesetze } & \multicolumn{2}{|c|}{ Prozent des BIP } & \multicolumn{2}{|c|}{ Prozent der Einnahmen } \\
\hline & \multicolumn{2}{|c|}{ Mittelwerte } & \multicolumn{2}{|c|}{ Mittelwerte } \\
\hline & 2 Jahre & 4 Jahre & 2 Jahre & 4 Jahre \\
\hline Revenue Act of 1978 & $-0,69$ & $-0,83$ & $-3,4$ & $-3,9$ \\
\hline Economic Recovery Tax Act of 1981 & $-1,91$ & $-2,89$ & $-9,0$ & $-13,3$ \\
\hline Tax Equity and Fiscal Responsibility Act of 1982 & 0,8 & 0,98 & 4,6 & 5,7 \\
\hline Social Security Amendments of 1983 & 0,2 & 0,21 & 1,1 & 1,2 \\
\hline Deficit Reduction Act of 1984 & 0,3 & 0,39 & 1,7 & 2,1 \\
\hline Tax Reform Act of 1986 & 0,22 & 0,01 & 1,2 & 0,1 \\
\hline Tax Relief Act of 1997 & $-0,08$ & $-0,14$ & $-0,4$ & $-0,7$ \\
\hline $\begin{array}{l}\text { Economic Growth and Tax Relief Reconciliation } \\
\text { Act of } 2001\end{array}$ & $-0,55$ & $-0,71$ & $-2,8$ & $-3,6$ \\
\hline Job Creation and Worker Assistance Act of 2002 & $-0,33$ & $-0,12$ & $-1,9$ & $-0,7$ \\
\hline $\begin{array}{l}\text { Jobs and Growth Tax Relief Reconciliation Act of } \\
2003\end{array}$ & 0,92 & 0,48 & $-4,8$ & $-2,5$ \\
\hline Economic Stimulus Act of 2008 & $-0,55$ & $-0,24$ & $-0,31$ & $-0,13$ \\
\hline Bank Bailout Bill of 2008 & $-0,36$ & $-0,19$ & $-0,22$ & $-0,12$ \\
\hline $\begin{array}{l}\text { American Recovery and Reinvestment Tax Act of } \\
2009\end{array}$ & $-0,94$ & $-0,52$ & $-0,58$ & $-0,32$ \\
\hline $\begin{array}{l}\text { Tax Relief, Unemployment Insurance } \\
\text { Reauthorization, and Job Creation Act of } 2010\end{array}$ & $-2,4$ & $-1,31$ & $-13,6$ & $-7,4$ \\
\hline American Taxpayer Relief Act of 2012 & $-1,8$ & $-1,78$ & $-8,5$ & $-8,4$ \\
\hline
\end{tabular}

Quellen: Tempalski 2006, S. 17; Tempalski 2013. 
Tabelle 2: Geschätzte Einnahmeausfälle der Kernbestandteile des ERTA

\begin{tabular}{lrrrrr}
\hline Gesetzeskomponenten & 1982 & 1983 & 1984 & 1985 & 1986 \\
\hline Einkommensteuer & & & & & \\
Steuersatzsenkungen & & & & & \\
Erleichterung für Doppelverdiener & $-25,8$ & $-65,7$ & $-104,5$ & $-122,7$ & $-143,8$ \\
Indexierung & $-0,4$ & $-4,4$ & $-9,1$ & $-11,0$ & $-12,6$ \\
Gesamt & - & - & - & $-12,9$ & $-35,8$ \\
(Neuschätzung 1983) & $-27,1$ & $-72,9$ & $-118,9$ & $-153,9$ & $-204,7$ \\
& $(-28,9)$ & $(-68)$ & $(-105,2)$ & $(-126,5)$ & $(-155,3)$ \\
Unternehmenserleichterungen & & & & & \\
Accelerated Cost Recovery System (ACRS) & $-9,6$ & $-16,8$ & $-26,3$ & $-37,3$ & $-52,8$ \\
F\&E Kredit & $-0,4$ & $-0,7$ & $-0,9$ & $-0,8$ & $-0,5$ \\
Gesamt & $-10,7$ & $-18,7$ & $-28,4$ & $-39,4$ & $-54,7$ \\
(Neuschätzung 1983) & $(-9,2)$ & $(-17,2)$ & $(-25,7)$ & $(-34,5)$ & $(-42,5)$ \\
& & & & & \\
Gesamt ERTA & $-37,7$ & $-92,7$ & $-150,0$ & $-199,2$ & $-267,7$ \\
(Neuschätzung 1983) & $(-38,4)$ & $(-87,8)$ & $(-134,8)$ & $(-166,6)$ & $(-205,2)$ \\
\hline
\end{tabular}

Quelle: Reproduziert aus Minarik 1990, 25.

Zentral für die sozialwissenschaftliche Debatte über die Genese des ERTA ist, dass das Ausmaß der Steuerkürzungen von 1981 selbst die Erwartungen von Befürwortern übertraf. Für Jacob Hacker und Paul Pierson stellt der ERTA das Paradebeispiel für Winner-take-all Politics dar. Demnach hätten verhältnismäßig undurchsichtige Netzwerke aus Spitzenverdienern, Unternehmen, Lobbyisten und politischen Eliten mit der Durchsetzung des ERTA an Mehrheitspräferenzen und demokratischen Wahlen vorbei agiert und ihre Interessen parteiübergreifend verankert. ${ }^{27}$

Insbesondere die veränderten Abschreibungsmöglichkeiten für Investitionen (ACRS und ITC) führten nach Verabschiedung des ERTA zu massiven Steuernachlässen für Unternehmen. In einem aufsehenerregenden Bericht zeigte die NonprofitOrganisation Citizens for Tax Justice, dass aus einer Stichprobe von 250 großen profitablen Unternehmen 128 zwischen 1981 und 1983 keine oder negative Unternehmenssteuern entrichteten. ${ }^{28}$ Die vielzitierte Untersuchung zeigte, dass Ronald Reagans früherer Arbeitgeber, General Electric, seit dem ERTA zwar 6,5 Milliarden US-Dollar Profite gemeldet hatte, aber nicht nur keine Unternehmenssteuern mehr zahlte, sondern sich 283 Millionen US-Dollar erstatten ließ. Diese und ähnliche Auswüchse des ERTA haben ihn in der Forschungsliteratur als Sündenfall unter-

27 Hacker, Pierson 2010, S. 133-134.

28 McIntyre, Folen 1984. 
nehmensfreundlicher Steuerpolitik erscheinen lassen, mit dem das amerikanische Steuersystem zu einer Art ungeschützter Allmende gut vernetzter Eliten verkommen war - Klientelpolitik der schlimmsten Sorte «. 29

Eine Schwäche derartiger elitenbasierter Erklärungen der Durchsetzung des ERTA ist, dass die politischen Prozesse, die sie begleiteten, alles andere als still und leise waren. ${ }^{30}$ Konflikte um den ERTA dominierten die wirtschaftspolitische Debatte nach der Wahl Ronald Reagans, und zumindest für einige Zeit war der Steuerschnitt in der Öffentlichkeit vergleichsweise populär. Auf dieser Basis hat insbesondere Monica Prasad die elitenbasierten Einordnungen des ERTA infrage gestellt. ${ }^{31}$ Auf Basis neu erschlossenen Archivmaterials argumentiert sie, dass die Reagan-Administration die Einkommensteuersenkungen zusammen mit hochrangigen Mitgliedern der Republikanischen Partei aus wahlstrategischen Motiven gegen die Interessen von Eliten durchgesetzt habe. Prasad zeigt, dass Unternehmensvertreter, wichtige Kongressabgeordnete und Regierungsvertreter nie wirklich an Arthur Laffers vielzitierte Rechtfertigung der Einkommensteuersenkung geglaubt haben, nach der sinkende Steuersätze zu steigenden Steuereinnahmen führen würden. Stattdessen befürchteten sie, dass eine massive Einkommensteuersenkung, wie sie der Reagan-Regierung vorschwebte, den Staatshaushalt ins Defizit befördern und die Inflation erneut anheizen könnte, und präferierten lediglich Steuersenkungen für Unternehmen und Spitzenverdiener. Prasad zeichnet nach, dass republikanische Reformer der Unternehmenslobby stückweise die Unterstützung für generelle Einkommensteuersenkungen abtrotzten und ihr obendrein ein Jahr nach Verabschiedung des ERTA Teile der im Gegenzug gewährten Investitionserleichterungen wieder entzogen. ${ }^{32}$

Dieser Lesart nach war der ERTA dem politischen Kalkül der Republikanischen Partei geschuldet, mit radikalen Steuersenkungen Mehrheiten zu gewinnen. Folgt man der dokumentierten Kommunikation zwischen Kongress und Reagan-Administration, war diese Taktik nicht von langer Hand geplant, sondern entstand stückweise aus dem Ringen um die Steuerreform mit den Demokraten im Kongress, den Reaktionen auf Wählerbefragungen und der internen Richtungssuche in der Republikanischen Partei nach Watergate. ${ }^{33}$

Prasads Analyse des ERTA legt wichtige Determinanten der Steuerreform frei. Tatsächlich war der Einkommensteuerschnitt, gemessen an statischen Einnahmeverlusten, mehr als dreimal so groß wie die Unternehmenssteuersenkungen über ACRS, ITC und Forschungs- und Entwicklungskredit (siehe Tabelle 2). ${ }^{34}$ Ebenso

29 Martin 1990, S. 31; Übersetzung T.E.

30 Culpepper 2010.

31 Prasad 2018.

32 Ebd., S. 141.

33 Ebd., S. 140.

34 Man sollte relativierend hinzufügen, dass Unternehmen mit den neuen Abschreibungsmöglichkeiten wesentlich »kreativer « umzugehen wussten als von der Politik vorhergesehen, weshalb die Schätzungen der Einnahmeausfälle problematisch sind. Da sich die Zeit nicht erneut ohne das ACRS betrachten lässt, ist eine Schätzung der tatsächlichen Einnahmeausfälle unmöglich. 
zutreffend ist die Beobachtung, dass die Regierung Teile der neuen Abschreibungsmöglichkeiten 1982 mit dem Tax Equity and Fiscal Responsibility Act zurücknahm, um das wachsende Haushaltsdefizit einzudämmen. Wichtig ist Prasads Nachweis, dass die Republikanische Partei in der Durchsetzung des ERTA einen fundamentalen Wandel durchmachte. Die Partei des ausgeglichenen Staatshaushalts und der Austerität verwandelte sich in die Partei der Steuersenkungen ohne Rücksicht auf Verluste. Prasads Analyse des ERTA ist Teil einer breiteren Bewegung in den amerikanischen Sozialwissenschaften, die Staatsfinanzen soziologisch untersucht, der New Fiscal Sociology. ${ }^{35}$ Im Vergleich mit den oben diskutierten Diffusionsmodellen neoliberaler Steuerreformen legt diese Literatur ein wesentlich stärkeres Gewicht auf kontingente historische Prozesse in der Untersuchung der Struktur und des Wandels von Steuersystemen. Ähnlich zu Prasads Argument zur Genese des ERTA diagnostiziert die neuere Fiskalsoziologie eine für die USA spezifische strategische Wandlung der Republikanischen Partei seit den frühen 1980er Jahren, mit radikalen Einkommensteuerschnitten Wählerstimmen zu gewinnen. ${ }^{36}$ Symbolpolitische Programme radikaler Steuersenkungen haben der Republikanischen Partei dieser Lesart nach zu einer erneuerten koalitionären Basis von individualistischen und konservativ-christlichen Wertvorstellungen mit Wirtschaftsinteressen verholfen.

Die Analysen der neueren Fiskalsoziologie sind unzweifelhaft äußerst wertvoll für das Verständnis politisch-gesellschaftlichen Wandels in den USA seit den 1970er Jahren. Teilweise nur gehen sie mit ihren Versuchen ein Stück zu weit, die neuere amerikanische Steuergeschichte als populistisch-sozialpolitisches Großexperiment darzustellen. Die symbolpolitische Kraft von Steuersenkungen schien sich nur wenige Monate nach Verabschiedung des ERTA erschöpft zu haben, als die Reagan-Regierung angesichts des wachsenden Haushaltsdefizits mit der Arbeit an einer Serie von Steuererhöhungen begann (siehe Tabelle 1). Und dass die Steuerpolitik der 1980er Jahre insbesondere im Unternehmenssektor wild zwischen verschiedenen Maßnahmen schwankte, muss nicht unbedingt bedeuten, dass die Unternehmenserleichterungen des ERTA lediglich taktisch eingesetzt wurden. Auch wenn diese seit 1982 zum Teil zurückgenommen wurden, boten sie den kapitalintensiven Segmenten der amerikanischen Industrie eine ansehnliche Beihilfe. Mehr noch: Zwischen den späten 1970er Jahren und dem Ende der 1980er Jahre wurde jede Steuerreform an zentraler Stelle mit Problemen der amerikanischen Industrie begründet - obwohl beträchtliche Verwirrung darum herrschte, wie genau eine wachstumsfreundliche Fiskalpolitik auszusehen habe. So erklären sich die Kontinuitäten zwischen Ronald Reagans und Jimmy Carters früherer Steuerpolitik sowie die Tatsache, dass die Unternehmensanreize des ERTA 1981 beträchtliche parteiübergreifende Unterstützung fanden.

Eine der wenigen Forschungsarbeiten, die industriepolitische Erwägungen ins Zentrum der Analyse des Aufkommens neoliberaler Steuerreformen in den USA rückt, ist Cathie Jo Martins historische Arbeit zum Wandel steuerpolitischer Leitvorstellungen in der Reagan-Administration. ${ }^{37}$ Martins grundlegendes Argument

35 Siehe überblicksweise: Martin et al. 2009.

36 Block 2009; Martin 2008.

37 Martin 1990. 
ist, dass Reagans Steuerpolitik in der Auseinandersetzung mit populären Interpretationen der Malaise der amerikanischen Wirtschaft seit Ende der 1960er Jahre entstand. Spezifischer weist sie nach, dass die Strukturen der Steuerreformen 1981 und 1986 ganz bestimmte Segmente der amerikanischen Industrie zu stärken versuchten. Während die erweiterten Abschreibungsmöglichkeiten von 1981 auf die Stärkung kapitalintensiver »alter " Industrien zielten, waren spätere Reformansätze an den Bedürfnissen »neuer ", forschungsintensiver Sektoren orientiert. Die Unternehmenskomponenten beider Steuerreformen waren von industriepolitischen Erwägungen durchzogen, die Wirtschaftswachstum und Exportstärke schaffen sollten, ohne dass der Staat unmittelbar industriepolitisch tätig werden musste.

»Die Steuerreform war auch durch den Bedarf der Republikanischen Partei motiviert, Alternativen zu industriepolitischen Vorschlägen der Demokraten zu finden. Industriepolitik sollte Ressourcen in schnell wachsende Sektoren lenken. Die Republikaner hatten ähnliche Ziele, wollten aber die Rolle des Staates minimieren. Steuerreformen boten einen Mechanismus, Ressourcen umzuverteilen, ohne dass die Regierung unmittelbar Einfluss nehmen musste. ${ }^{38}$

Anders als von Prasad interpretiert, müssen die Richtungswechsel in der Unternehmensbesteuerung damit nicht zwingend auf Richtungswechsel in der Wirtschaftsfreundlichkeit der Regierung hindeuten. Vielmehr lassen sie sich als Ausdruck einer fortwährenden Debatte verstehen, wo zukünftige Quellen industrieller Revitalisierung liegen und wie sich diese Quellen stärken lassen könnten. Hier wie an anderen Stellen war die Reagan-Regierung, was ihre politischen Rezepte anging, wesentlich weniger linientreu, als Theorien ideellen, paradigmatischen Wandels vermuten lassen. Im Prozess der Durchsetzung und Einführung von Policies suchte sie pragmatisch nach Koalitionen für ihre Politik.

Mein Argument schließt an genau diese Deutung von Reagans Steuerreformen an. Die Beschwörung des kommenden Niedergangs der amerikanischen Industrie gab neoliberalen Reformern ein weites Handlungsfenster, in dem sich pfadbrechende Reformen durchsetzen lassen konnten, und diese Durchsetzungsstrategien prägten wiederum, wie die jeweiligen Reformen schlussendlich aussahen.

\section{Niedergangserzählungen und die Steuerpolitik der Reagan-Regierung}

Historiker haben die Besonderheiten der Rhetorik Ronald Reagans gerade in der Abkehr vom schweren, pflichtbewussten und zukünftige Gefahren beschwörenden Ton Jimmy Carters verortet. ${ }^{39}$ Carters Crisis of Confidence habe Reagan sein Morning again entgegengesetzt - und damit der beschwerlichen eine offene Zukunft. In der Steuerpolitik - dem legislativen Erfolg der Regierung - galt das nicht. Sowohl in seiner Wahlkampagne als auch in seiner Inaugurationsrede griff Reagan genau die Krisendiagnosen auf, die Nixon, Ford, Carter und den Kongress seit Anfang der 1970er Jahre beschäftigten. Die allgemeine Malaise der amerikanischen Wirtschaft befeuerte eine Debatte um den drohenden Niedergang der ameri-

38 Ebd., S. 31; Übersetzung T.E.

39 Rodgers 2011.

Leviathan, 47. Jg., 2/2019 
kanischen Industrie, mit der die Regierung ihre Steuersenkungen rechtfertigte und auf deren Basis sie sie schließlich durchsetzen konnte.

Tabelle 3: Ausgewählte makroökonomische Indikatoren der USA, 1971-1988

\begin{tabular}{|c|c|c|c|c|}
\hline & $\begin{array}{r}\text { Änderung des } \\
\text { Bruttoinlandsprodukts }\end{array}$ & $\begin{array}{r}\text { Änderung der } \\
\text { Arbeitsproduktivität }\end{array}$ & $\begin{array}{r}\text { Änderung des } \\
\text { Preisniveaus }\end{array}$ & Arbeitslosenquote \\
\hline 1971 & 3,3 & 3,9 & 5,1 & 6,0 \\
\hline 1972 & 5,3 & 3,4 & 4,3 & 5,6 \\
\hline 1973 & 5,6 & 3,1 & 5,5 & 4,9 \\
\hline 1974 & $-0,5$ & $-1,6$ & 9,0 & 5,6 \\
\hline 1975 & $-0,2$ & 2,7 & 9,3 & 8,5 \\
\hline 1976 & 5,4 & 3,5 & 5,5 & 7,7 \\
\hline 1977 & 4,6 & 1,7 & 6,2 & 7,1 \\
\hline 1978 & 5,5 & 1,4 & 7,0 & 6,1 \\
\hline 1979 & 3,2 & $-0,2$ & 8,3 & 5,9 \\
\hline 1980 & $-0,3$ & 0,0 & 9,1 & 7,2 \\
\hline 1981 & 2,5 & 1,6 & 9,4 & 7,6 \\
\hline 1982 & $-1,8$ & $-0,9$ & 6,2 & 9,7 \\
\hline 1983 & 4,6 & 4,2 & 3,9 & 9,6 \\
\hline 1984 & 7,2 & 2,2 & 3,6 & 7,5 \\
\hline 1985 & 4,2 & 1,7 & 3,2 & 7,2 \\
\hline 1986 & 3,5 & 3,0 & 2,0 & 7,0 \\
\hline 1987 & 3,5 & 0,6 & 2,5 & 6,2 \\
\hline 1988 & 4,2 & 1,6 & 3,5 & 5,5 \\
\hline
\end{tabular}

Quelle: US Bureau of Labor Statistics und Bureau of Economic Analysis.

Die wirtschaftliche Lage der USA war im Jahr 1981 derart vertrackt, dass unmittelbar nach Amtsantritt erheblicher Handlungsdruck auf der Reagan-Regierung lastete. Die amerikanische Ökonomie war im Jahr 1980 leicht geschrumpft, litt unter 9,1 Prozent Inflation und einer Arbeitslosenquote von 7,2 Prozent, während die Entwicklung der Arbeitsproduktivität stagnierte (siehe Tabelle 3). Zur Irritation vieler Beobachter wandelte sich der lange für natürlich gehaltene Handelsbilanzüberschuss der amerikanischen Wirtschaft Anfang der 1970er Jahre in ein stetig wachsendes Defizit (siehe Abbildung 1). Zu den makroökonomischen Turbulenzen gesellten sich Anzeichen mikroökonomischer Verwerfungen. Um nur zwei Beispiele zu nennen: Chrysler musste Ende 1979 vom Staat gerettet werden, während die 
amerikanische Stahlindustrie bis Mitte 1982 nach zeitgenössischen Schätzungen ein Drittel ihrer 400.000 Angestellten abbaute. ${ }^{40}$

\section{Abbildung 1: Entwicklung des Handelsbilanzsaldos der USA, 1960-1990}

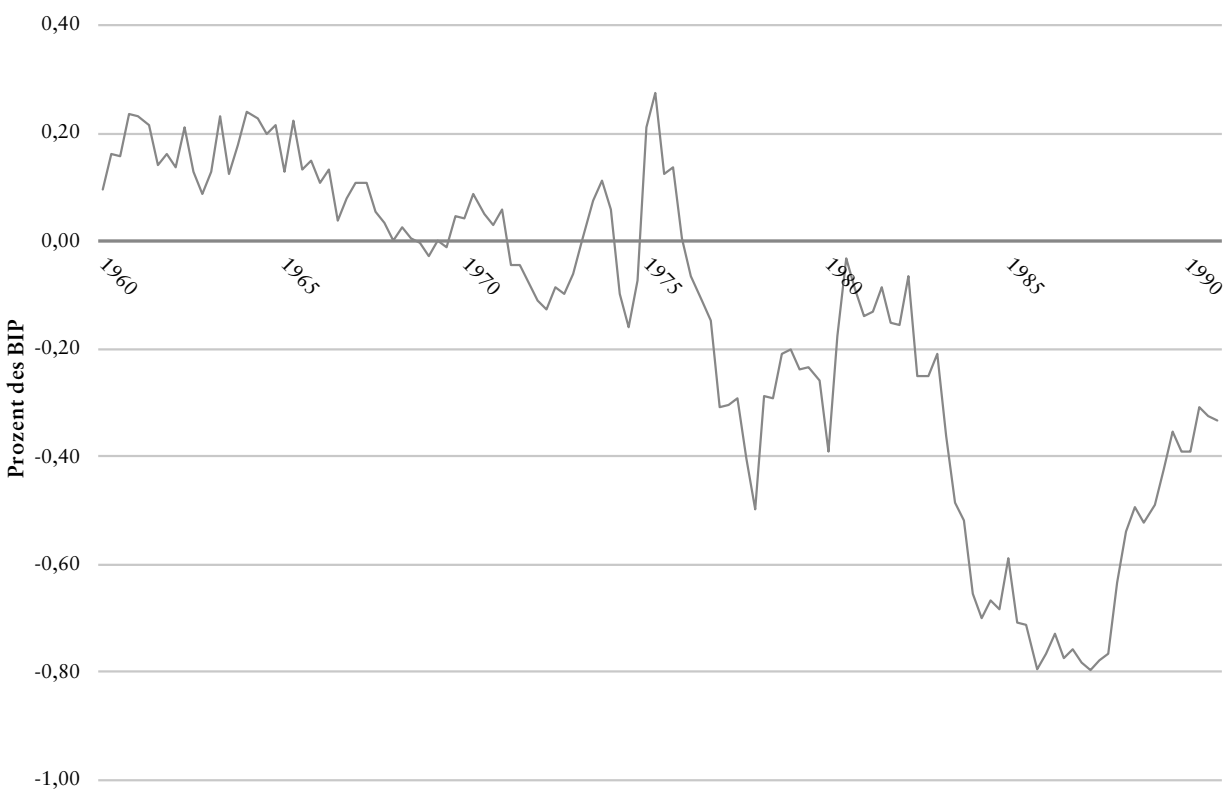

Quelle: United States Burean of Economic Analysis.

Diese wirtschaftlichen Verwerfungen befeuerten eine Debatte über die wirtschaftliche Zukunft der USA, die Otis Graham die »Great Industrial Policy Debate « genannt hat. ${ }^{41}$ Politiker, Wirtschaftsvertreter und Intellektuelle diskutierten, inwieweit die wirtschaftlichen Probleme der Zeit neuartiger politischer Eingriffe bedürften. Das Time Magazine titelte am 30. März 1981 mit »How Japan does it: the world's toughest competitor " und griff damit einen Ton auf, der immer weitere Kreise im wirtschaftspolitischen Diskurs der Zeit zog. Jimmy Carter war in den letzten Jahren seiner Amtszeit daran gescheitert, der Malaise sektorpolitisch zu begegnen, und verlegte seinen Fokus mit der Berufung Paul Volckers zum Chef der Federal Reserve auf die Bekämpfung der Inflation. ${ }^{42}$ Dennoch griff auch er regelmäßig auf die neue Sprache ökonomischer Kommentatoren zurück, die die amerikanische Wirtschaft in einer existentiellen Konkurrenz mit aufstrebenden kapitalistischen Nationen sahen. Als Teil einer gescheiterten Initiative, privatwirtschaftliche Innovationen zu fördern, ermahnte er den Kongress im Jahr 1979, dass

40 Borrus 1983; Hoerr 1988.

41 Graham 1992.

42 Ebd., S. 44. 
die zunehmende internationale Konkurrenz eine aktivere Rolle des Staates in der Industrieförderung notwendig machen würde. ${ }^{43}$

Im Vergleich mit den Äußerungen vieler Intellektueller waren Carters Warnungen noch gemäßigt. Der Wirtschaftshistoriker Charles Kindleberger fragte 1973, ob die amerikanische Industrie ein Muster wiederhole, das die britische Industrie in der Konkurrenz mit dem Deutschen Reich und den USA Ende des 19. Jahrhunderts erlebt hatte - »verursacht von einer analogen Herausforderung durch Japan «. ${ }^{44}$ Ein Protagonist der Debatte zum Niedergang der amerikanischen Industrie, Lester Thurow, beobachtete das einbrechende Produktivitätswachstum der US-Wirtschaft und warnte, dass »es nur eine Frage der Zeit ist, bis wir relativ rückständig sind. Wenige wichtige Länder sind an ausländischen Feinden gescheitert; viele sind wegen ihrer internen Probleme untergegangen ${ }^{.} .5$

Viele Protagonisten der Debatte um eine amerikanische Industriepolitik forderten für die USA untypische Schritte, wie etwa die Einrichtung eines investitionslenkenden »Industrieministeriums «. Obwohl sie wiederholt in internen Debatten der Demokratischen Partei aufgegriffen wurden, hatten derartige planerische Vorstöße nie wirklich eine Chance, politische Wirklichkeit zu werden. Stattdessen sind Bestandteile der ökonomischen Niedergangsinterpretation in etliche Policies hinein diffundiert. Gute wirtschaftspolitische Beispiele für diesen Prozess sind das Patentund Kartellrecht sowie die Stärkung der Rechte von Shareholdern seit Ende der 1970er Jahre. ${ }^{46}$ In der Außenpolitik entwickelte sich seit den späten 1960er Jahren eine lebhafte Debatte um einen drohenden Hegemonic Decline - die Sorge, dass die Vereinigten Staaten ihre Hegemonie in der globalen Weltordnung verlieren würden. Seit den späten 1970er Jahren bekam diese Debatte eine zunehmend politisch-ökonomische Färbung. Nachlassendes Produktivitätswachstum, gesellschaftliche Dekadenzerscheinungen und eine abnehmende Bereitschaft, für globale öffentliche Güter aufzukommen, würden den Weltmachtstatus der USA unterwandern. ${ }^{47}$ Wie nicht selten in den Vereinigten Staaten bekamen wirtschaftspolitische Entscheidungen mit diesen Debatten eine außen- und sicherheitspolitische Dringlichkeit.

In der Steuerpolitik wurde vor allem die Sorge um den Einbruch des Produktivitätswachstums diskutiert. Zuvorderst Wirtschaftsvertreter aus dem produzierenden Gewerbe drängten seit den frühen 1960er Jahren auf eine erweiterte Förderung von Kapitalinvestitionen im Steuerrecht. ${ }^{48}$ Seit dem Revenue Act von 1971 waren sie zunehmend erfolgreich, steuerliche Beihilfen über die Veränderung von Abschreibungsregeln durchzusetzen. Die Nixon-Regierung etwa versprach:

»Das wichtigste, das dieses Gesetz bedeutet, sind Arbeitsplätze. [...] Der [investment tax credit] wird Arbeitsplätze schaffen, indem er Unternehmen zur Anschaffung neuer Anlagen

43 Carter 1979.

44 Kindleberger 1973; Übersetzung T.E.

45 Thurow 2001 [1980], S. 77; Übersetzung T.E.

46 Christophers 2016; Dobbin, Jung 2010; Ergen, Kohl 2019.

47 Gilpin 1981; Kennedy 1988.

48 Fisher 1985. 
motiviert, und er wird, indem er die Produktivität unserer Arbeiter erhöht, dazu beitragen, amerikanische Unternehmen global wettbewerbsfähiger zu machen und dementsprechend mehr Arbeitsplätze hier in den USA zu halten. « ${ }^{49}$

Das Muster, mit Verweisen auf die internationale Konkurrenz auch Kräfte für Steuersenkungen zu mobilisieren, die traditionell ein stark progressives Steuerregime unterstützten, wiederholte sich über die folgenden Jahrzehnte immer wieder. Charls Walker, 1971 noch Angestellter des Finanzministeriums und in der Folge einer der einflussreichsten Lobbyisten in der amerikanischen Steuergesetzgebung, hat in einer Gesetzesbiografie zusammengefasst, dass die Unternehmensanreize 1971 vor allem dadurch im Kongress durchgesetzt wurden, dass die amerikanischen Gewerkschaften und ihre Kongresskontakte mit Verweis auf die internationale Konkurrenz für diesen Schritt zu mobilisieren waren:

»Den Kongress im Hinblick auf die internationale Konkurrenz zu erziehen war der Schlüssel. Die [Gewerkschaftsbewegung], die damals noch sehr mächtig im Kongress war, hatte sich von ihrer traditionellen Freihandelsposition zum Protektionismus bewegt; im Kongress herrschte Aufregung um die internationale Konkurrenz und den `Export von Arbeitsplätzen [...]. Kein Wort wurde darüber verloren, ob die Steuererleichterungen für Unternehmen fair waren oder nicht. « ${ }^{50}$

Was diese Dynamik Ende der 1970er Jahre noch verstärkte, war die Verschärfung der Krise amerikanischer Fertigungsindustrien und eine aus heutiger Sicht absurd wirkende Debatte über eine drohende Kapitalknappheit. Blyth hat diese Debatte treffend eine »parlamentarische Obsession der späten 1970er Jahre " genannt. ${ }^{51}$ Seit 1974 versuchten die verschiedensten Institutionen auszurechnen, ob die in den USA verfügbaren Ersparnisse genügen würden, um den Kapitalbedarf der amerikanischen Wirtschaft für das nächste Jahrzehnt zu decken. Die New York Stock Exchange errechnete 1974 eine Lücke von 650 Milliarden US-Dollar bis 1985, Finanzminister William Simon wahlweise 2,5 bis 4,5 Billionen Dollar. ${ }^{52}$ Das zentrale Argument der Debatte zum Kapitalengpass war, dass sich die wirtschaftlichen Probleme der USA im Kern auf einen Einbruch des Produktivitätswachstums zurückführen ließen. Dieser wiederum sei durch zurückgehende Kapitalinvestitionen verursacht, die von einer unzureichenden Sparquote gedämpft worden seien. ${ }^{53}$ Und auch ein akademisch hoch angesehener Ökonom wie Martin Feldstein, der später Vorsitzender des ökonomischen Beraterstabs Reagans wurde, ließ sich auf Geschichten über den früheren »Niedergang « Großbritanniens ein, um dieser Krisendiagnose Nachdruck zu verleihen:

»Wir haben eine sehr niedrige Sparquote. [...] Der langfristige Effekt ist, dass wir weniger produktiv sein werden. Blicke ich auf die europäischen Volkwirtschaften und sehe, was mit Ländern wie England passiert ist, das mit ansehen muss, wie es von Ländern mit höheren Sparquoten überholt wird, habe ich eine Vorahnung der Art von Zukunft, in der wir uns

49 Nixon 1971; Übersetzung T.E.

50 Fullerton et al. 1994, S. 215, Fußnote 7; Übersetzung T.E.

51 Blyth 2002, S. 161; Übersetzung T.E.

52 Eisner 1977.

53 Gute Zusammenfassungen dieses Arguments sind die Stellungnahmen der Ökonomen Feldstein und Meiselman in: US Congress 1977. 
wiederfinden könnten und in der wir ein relativ zu anderen Ländern ärmer und ärmer werdendes Land sind. $«^{54}$

Die Aufregung um die amerikanische Sparquote war eine ökonomische und in Teilen konsumkritische Variante verbreiteter konservativer Zeitdiagnosen der 1970er Jahre, nach denen reiche Nachkriegsgesellschaften das Maßhalten verlernt hätten. ${ }^{55}$ Reagan begann seine Inaugurationsrede mit der Krisendiagnose, man habe über Jahrzehnte "unsere Zukunft und die unserer Kinder" der »kurzweiligen Bequemlichkeit der Gegenwart « geopfert und »über seine Verhältnisse gelebt «. ${ }^{56}$ Ihrem Wirtschaftsprogramm stellte die Regierung voran: »Wir können die Anreize für Arbeit und Sparsamkeit wiederbeleben. Wir können den Willen zu privaten Investitionen wiederherstellen, die uns einen stetig steigenden Lebensstandard erlauben. $«^{57}$ Abseits von intern selten ernst genommenen Argumenten zur Aufkommensneutralität eines Einkommensteuerschnitts - wie die vieldiskutierte Laffer-Kurve bestand die verbindende Rechtfertigungsgrundlage der verschiedenen Komponenten des ERTA in der kollektiven Herausforderung, Sparsamkeit und Investitionstätigkeit wiederherzustellen.

Blickt man in die Verhandlungen zum ERTA - oder zu »Kemp-Roth «, wie er vor seiner Verabschiedung oft genannt wurde -, bezweifelten die wenigsten Experten und Interessengruppen die prinzipielle Logik des Gesetzesvorschlags, nach der die Gesundung der amerikanischen Ökonomie wesentliches Ziel des Steuerrechts sei und es einer regressiven Reform bedürfe. Einer der häufig genannten Kritikpunkte, etwa von Vertretern des Kleinunternehmertums, war die Sorge, dass ein Steuernachlass - gleich welcher Art - inflationär wirken würde. ${ }^{58}$ Gewerkschaftsvertreter waren unter den wenigen Angehörten, die auf die Gerechtigkeitsprobleme einer offen regressiven Steuerreform aufmerksam machten. Wenngleich sie noch stark den industriepolitischen Initiativen Jimmy Carters nachtrauerten und staatlich gelenkte Hilfen für kriselnde Unternehmen und Regionen forderten, bestritten auch sie das grundlegende Desiderat nicht, die amerikanische Industrie steuerbasiert zu rekapitalisieren. ${ }^{59}$ Besonders bestimmt setzten sich Vertreter der klassischen Industrieregionen im Rust Belt für Investitionserleichterungen ein. Politische Verbündete der Stahlbranche etwa, organisiert im Congressional Steel Caucus, zeichneten das Bild einer brachliegenden heimischen Industrie, die drohe, von internationalen Konkurrenten ausgelöscht zu werden. »Stahl ist das fundamentale Element, der Grundpfeiler, der Achsnagel einer florierenden Nation «, beteuerte ein Abgeordneter aus Pennsylvania, »jede Million Tonnen Importstahl bedeutet 5.000 verlorene amerikanische

54 Ebd., S. 174; Übersetzung T.E.

55 Krippner 2012; Schäfer 2008.

56 Reagan 1981; Übersetzung T.E.

57 The White House 1981, S. 1-2; Übersetzung T.E.

58 Siehe etwa die Stellungnahmen in US Congress 1981, Third Session; vgl. auch die früheren Diskussionen der Carter-Regierung in Hutcheson 1977.

59 US Congress 1981, Second Session, S. 584-588. 
Arbeitsplätze. ${ }^{60}$ Genau diese Saite bespielte die Reagan-Regierung in der Bewerbung ihrer Reform als Reindustrialisierungsprogramm. Der Direktor des Office of Management and the Budget, David Stockman, versprach in Reaktion auf eine Frage zu den industriellen Krisen im Rust Belt, dass ACRS und ITC die andauernde Welle aus Werksschließungen und Verlagerungen beenden würden:

»Wenn man die Politik so anpassen kann, dass [die Industrie] ihre Investitionen schneller abschreiben und alte Maschinen und Anlagen ersetzen oder einige der großen, integrierten Fabriken restrukturieren kann [...], haben sie eine wesentlich bessere Chance, nicht nur gegenüber dem Südwesten, sondern auch dem Rest der Welt wettbewerbsfähig zu sein. Sollten wir diese neuen Abschreibungsmöglichkeiten nicht implementieren, fürchte ich, dass die Fabriken abwandern werden, aber nicht nach Oklahoma oder Texas, sondern in andere Länder. « ${ }^{61}$

Düstere Prophezeiungen wie diese waren sicher nicht der einzige Grund, weshalb die Reagan-Regierung es schaffte, den größten Steuerschnitt der modernen amerikanischen Geschichte in einem demokratisch dominierten Repräsentantenhaus durchzusetzen. Sie helfen aber zu erklären, wie eine offen regressive Reform sich als selbstverständliche Reaktion auf die Probleme der Zeit darstellen lassen konnte. Wie von Prasad und Elitentheorien neoliberaler Steuerpolitik beschrieben, fand um den ERTA eine wahre Lobbying-Schlacht statt, in der einzelne Kongressabgeordnete stückweise zur Unterstützung des Gesetzespakets gebracht wurden. Ohne anerkannte Rechtfertigung allerdings, dass Steuerschnitte im gesellschaftlichen Gemeininteresse waren, ist die politische Gräben überspannende Unterstützung des ERTA schwer zu erklären.

\section{Die handelspolitischen Konsequenzen einer Politik leerer Versprechen}

Selbst wenn sie über das Jahr 1982 hinaus beibehalten worden wären, waren die Revitalisierungsmittel des ERTA, verglichen mit den Problemen der amerikanischen Industrie, von kläglichem Ausmaß. ${ }^{62}$ Insbesondere die Zinspolitik der Federal Reserve, die der amerikanischen Gesellschaft mit Leitzinssätzen von bis zu 20 Prozent die Inflation auszutreiben versuchte, verschärfte die Krise der amerikanischen Industrie. Abgesehen von der tiefen Rezession, in die die Zentralbank die amerikanische Wirtschaft stürzte, wirkte sich ihre Zinspolitik gravierend auf den Wechselkurs des Dollar und damit auf das amerikanische Handelsbilanzdefizit aus. Der Rat der Wirtschaftsberater (Council of Economic Advisers) hat 1983 geschätzt, dass der US-Dollar seit 1979 real 53,8 Prozent gegen die Deutsche Mark aufgewertet hatte, 36,8 Prozent gegen den japanischen Yen und 50,8 Prozent gegen den französischen

60 US Congress 1981, Third Session, S. 2357-2358; Übersetzung T.E. Tatsächlich brachten demokratische Abgeordnete aus dem Mittleren Westen mit dem Konzept des Expensing wesentlich großzügigere Erleichterungen für Unternehmen ins Gespräch, als sie die Reagan-Regierung forderte und der ERTA schließlich vorsah.

61 US Congress 1981, First Session, S. 79; Übersetzung T.E.

62 Eine gute international und sektoral komparative Übersicht industrieller Probleme der Zeit findet sich bei Dertouzos et al. 1991. 
Franc. ${ }^{63}$ Ferner zeigten alle gängigen Maße für Kapitalinvestitionen, dass der ERTA keinen ohne Weiteres ersichtlichen Effekt auf den amerikanischen Kapitalstock gehabt hatte. ${ }^{64}$

Die Verschärfung der Probleme der amerikanischen Industrie sorgte dafür, dass die Debatte, die die Durchsetzung des ERTA begleitet hatte, bis in die zweite Hälfte der 1980er Jahre nicht abebbte, sondern sich noch verschärfte. Insbesondere der Kongress war wirtschaftspolitisch bis Ende der 1980er Jahre von Versuchen bestimmt, industriellen Problemen politisch entgegenzuwirken. Die Demokratische Partei versuchte mehrmals, die industrielle Revitalisierung zum Kernthema der Vorwahlen (Primaries) und der Präsidentschaftswahlen 1984 zu machen. ${ }^{65}$ Gleichzeitig brachten Abgeordnete seit den späten 1970er Jahren verstärkt Gesetze in den Kongress ein, die die amerikanische Industrie handelspolitisch entlasten sollten. Schätzungen gehen davon aus, dass zwischen 1979 und 1984 jährlich um die 30 klar protektionistische Gesetzesvorlagen in den Kongress eingebracht wurden, zwischen 1985 und 1986 um die 45.66

Wirtschaftsliberale Kommentatoren kritisierten am »Protektionismus « der Reagan-Jahre, dass die Regierung dem Kongress mit bilateral verhandelten Importbeschränkungen, Liberalisierungsforderungen im Ausland und Zugeständnissen von Handelspartnern zuvorzukommen versuchte. Diese Abkommen zu einer Art »verwaltetem Handel « betrafen sowohl einzelne Produkte und Industrien als auch makroökonomische Zugeständnisse, etwa zur Wechselkursmanipulation. Noch Ende der 1980er Jahre hat Milton Friedman über die Handelspolitik der ReaganRegierung geurteilt, sie hätte Smoot-Hawley - das Symbol des amerikanischen Protektionismus der Zwischenkriegszeit - im Vergleich "absolut harmlos « aussehen lassen. ${ }^{67}$ Schon 1981 verlängerte die Regierung mit dem »Multifiber Agreement « Importquoten für Textilien und handelte »freiwillige Exportbeschränkungen « für PKWs aus Japan aus, die japanische Exporte auf ca. zwei Millionen Fahrzeuge pro Jahr begrenzten (und die erst 1994 unter der Clinton-Regierung aufgehoben wurden). 1982 verhandelte sie Exportbeschränkungen für Stahl mit europäischen Ländern, die 1984 auf die Mehrzahl nichteuropäischer Exporteure ausgedehnt wurden. Im Jahr 1986 brachte man deutsche, japanische, schweizerische und taiwanesische Maschinenbauer zur freiwilligen Begrenzung von Exporten, verlängerte das »Multifiber Agreement « und zwang Japans Halbleiterindustrie, ihre Preise in Drittmärkten zu erhöhen und den Marktanteil amerikanischer Hersteller in Japan zu erweitern (was die japanische Regierung vor eine unlösbare Aufgabe stellte und unmittelbar weitere Handelssanktionen nach sich zog). 1988 begann die Regierung mit der Führung eines Entwicklungskartells für ausgewählte amerikanische Halbleiterhersteller, genannt »Sematech", mit dessen Hilfe sie ihre internationale Stellung sichern

63 Council of Economic Advisers 1983, S. 66.

64 Ebd., S. 80-81.

65 Siehe den Artikel »Drafting a democratic industrial plan «, in New York Times vom 28. August 1983, S. 31.

66 Richardson et al. 1994, S. 640.

67 Friedman 1987. 
sollten. Seit 1982 drängte die Regierung Reagans die japanische Regierung zu Maßnahmen, die den Yen gegenüber dem US-Dollar aufwerten sollten. Verhandlungen mit der Bundesrepublik und Japan über Währungsmanipulationen zogen sich über die gesamte erste Hälfte der 1980er Jahre. Im »Plaza-Agreement « von 1986 schließlich verpflichteten sich beide Länder zu beträchtlichen Aufwertungen gegenüber dem US-Dollar.

Diese und zahllose weitere Eingriffe der Reagan-Regierung sind in der historischen Literatur als Minimalreaktionen auf politischen Druck aus dem Kongress beschrieben worden. Im Ganzen sei die Regierung gerade davon abgerückt, heimische Industrien zu schützen, und habe den Fokus der Handelspolitik stattdessen auf die »Liberalisierung « ausländischer Volkswirtschaften gelenkt. ${ }^{68}$ Derartige Einordnungen übergehen erstens, dass der Begriff der »Liberalisierung " des Auslands erhebliche Möglichkeiten bot, Handelsbedingungen im eigenen Interesse durchzusetzen, und zweitens, dass die Regierung am politischen Druck aus dem Kongress nicht unschuldig war.

In einer Rede vor Industrievertretern und Kongressabgeordneten beschwichtigte Reagan die Anwesenden mit der Äußerung, »fairer Handel« sei Handel, der unter US-amerikanischen institutionellen Bedingungen zustande käme:

»Falls einige unserer Handelspartner ihre Exporteure auf eine Art unterstützen, die gegen unsere eigenen Gesetze verstoßen würde, dann ist keine Chancengleichheit mehr gegeben und es gibt keinen freien Handel. Falls einige unserer Handelspartner viel Geld für Forschung und Entwicklung in ihre Industrien stecken und für deren Kosten bürgen, müssen unsere Hersteller und Exporteure eine weitere Bürde tragen, und das ist kein freier Handel. $\ll^{69}$

Derartig offene Bestimmungen »fairen « Handels führten zu einer wahren Flut an wirtschaftlichen Beschwerden über ausländische Gesetze, Regelungen und Praktiken und darauf folgende Regierungsinterventionen zum Abbau ausländischer »Handelsbarrieren«. Ebenfalls 1985 machte Reagan Malcolm Baldridge, seinen wahrscheinlich am wenigsten dem Freihandel zugeneigten Minister, zum Vorsitzenden einer neuen »Strike Force", die eigenständig Barrieren für den Export amerikanischer Güter und Dienstleistungen im Ausland aufspüren sollte: »Ich werde nicht zusehen, wie amerikanische Arbeiter ihre Jobs verlieren, weil andere Nationen sich nicht an die Regeln halten ", gab Reagan zu ihrer Gründung bekannt. ${ }^{70}$

Äußerungen wie diese waren nicht bloß Ad hoc-Reaktionen auf gesellschaftliche Forderungen nach Handelsbeschränkungen, sondern Teil einer mit dem ERTA losgetretenen Kommunikationsfalle. Die Wirtschaftspolitik der Regierung sollte zu einer Gesundung der amerikanischen Industrie im internationalen Wettbewerb führen, was über die 1980er Jahre vor allem in Problemregionen und Problemindustrien nicht annähernd gelang. Beide Reagan-Regierungen setzten alles daran, Issueownership der wirtschaftlichen Gesundung des Landes zu behalten, ${ }^{71}$ scheiterten

68 Chorev 2007.

69 Reagan 1985, S. 4; Übersetzung T.E.

70 The White House 1985, S. 1; Übersetzung T.E.

71 Petrocik 1996. 
aber mit jeder ihrer Initiativen an strukturellen Umständen oder an der Implementierung ihrer Lösungsstrategien. Ihr vieldiskutiertes Konzept zur Revitalisierung von Problemregionen, die »Urban Enterprise Zones «, die über eine Reihe regulatorischer und steuerlicher Anreize Unternehmensansiedlungen fördern sollten, scheiterte über drei Jahre im Kongress. ${ }^{72}$ Ihre 1983 großangekündigte Commission on Industrial Competitiveness, die Unternehmenslenker, Regierungsvertreter und Arbeitnehmervertreter zur Entwicklung von Handlungsoptionen zusammenbrachte, entwickelte größtenteils Gemeinplätze, die von der Regierung obendrein in weiten Teilen zurückgewiesen wurden. ${ }^{73}$ Reagan war mit dem Versprechen angetreten, » diesen industriellen Riesen wiederauferstehen zu lassen $"{ }^{74}$ und seine Regierung hatte insbesondere in den Konflikten um den ERTA immer wieder versprochen, dass ihr Ansatz in der Wirtschaftspolitik die notwendige wirtschaftliche Trendwende bringen würde. Genau dieses Spiel mit industriellen Niedergangserzählungen erschwerte es ihr in der Folge, Rufe nach handelspolitischen Interventionen abzuwehren.

\section{Diskussion: Neoliberale Politik und Erzählungen über die Zukunft}

Karl Polanyi hat in seiner Großen Transformation argumentiert, dass liberale Reformer so besorgt um die reine Implementierung ihrer Lehren waren, dass sie Gesellschaften überforderten und schließlich weniger Liberalismus bekamen, als eine gemäßigte Politik bedeutet hätte. ${ }^{75}$ Auf eine Art lässt sich das Schicksal neoliberaler Reformen bis in die Gegenwart mit Polanyis Argument verstehen. Jene früheren industriellen Kernregionen, die schon Reagan in der Handelspolitik Probleme machten, weil sie sich beharrlich nicht »erneuern « wollten, gelten gegenwärtig als wesentliche Triebkräfte des Wiederauflebens protektionistischer und isolationistischer Rhetorik in den USA und in Großbritannien. ${ }^{76}$ In diesem Sinn gibt es in der Wirtschaftspolitik zwischen den republikanischen Regierungen in den 1980er Jahren und in der Gegenwart wesentlich mehr Kontinuitäten als gemeinhin angenommen.

Teilweise haben diese politischen Kontinuitäten eine tiefere gesellschaftliche Ursache. Wie auch in gegenwärtigen politischen Konflikten taten sich in der Steuer- und Handelspolitik der 1980er Jahre Spaltungen in der amerikanischen politischen Ökonomie auf, die typisch für postindustrielle Gesellschaftsordnungen sind. ${ }^{77}$

72 Carleson 1982.

73 Reagan 1983. Der Abschlussbericht wurde Anfang 1985 von allen wesentlichen Stellen der Regierung kommentiert und im Cabinet Council on Commerce and Trade beraten. $\mathrm{Zu}$ einer umfassenden Policy ist er nie geworden.

74 Reagan 1981; Übersetzung T.E.

75 Polanyi 2001 [1944]; ein verwandtes Argument aus der neueren politischen Ökonomie findet sich bei Katzenstein 1985 .

76 Für einen Einblick in die Debatte: McQuarrie 2017.

77 Beramendi et al. 2015. 
"Alte " Industrien und sie beherbergende Regionen stritten für Anpassungshilfen und Revitalisierungsmaßnahmen, während »neue « Industrien generelle Erleichterungen im Steuerrecht gerne mitnahmen, sich aber gegen "Diskriminierung " zugunsten kapitalintensiver Sektoren wandten. Dieser Konflikt wanderte stückweise in die Handelspolitik - mit dem Zusatz, dass sich unter Bedrängnis ihrer japanischen Konkurrenz zunehmend auch Vertreter »neuer « Industrien, etwa aus der Halbleiterei und Elektronik, mit dem »verwalteten Handel « der 1980er Jahre anfreundeten. Inwieweit auch in gegenwärtigen handelspolitischen Auseinandersetzungen »junge « Industrien den Druck der Regierung auf die Handelspartner der USA unterstützt haben, sollte der Beurteilung durch zukünftige historische Studien überlassen bleiben. Zumindest verweisen auch amerikanische Regierungen im 21. Jahrhundert auf Bedrohungsszenarien zur Zukunft junger Branchen, um die Struktur des Handels mit China als »weder natürlich noch nachhaltig « zu brandmarken. ${ }^{78}$

Durch einen genaueren Blick auf politische Durchsetzungsprozesse, wie er hier angedeutet wurde, lässt sich präziser ausdrücken, wie Liberalisierungsmaßnahmen handelspolitische Gegenbewegungen auslösen können. Es ist keinesfalls ungewöhnlich, dass politisch heikle Reformen mit Geschichten über zukünftige Gefahren und Versprechen ihrer Abwendung durchgesetzt werden. Die Beschwörung kollektiver Gefahren macht Koalitionen zwischen ansonsten widerstreitenden Interessen möglich. Genau dies ließ sich bei der Durchsetzung des ERTA beobachten. Am Ende des Jahres 1981 gab es beinahe keine Interessengruppe mehr, die sich prinzipiell gegen die steuerliche Förderung der Kapitalakkumulation stellte. Die Versprechen der Regierung auf industrielle Revitalisierung führten dazu, dass über die nächsten Jahre immer wieder von ihr verlangt wurde, die amerikanische Industrie im internationalen Wettbewerb zu unterstützen.

Anhand der Durchsetzung des ERTA lässt sich ferner zeigen, dass Erwartungen über die Zukunft Teil politischer Durchsetzungsstrategien und Gegenstand politischer Konflikte sein können. ${ }^{79}$ Gemessen an ihren wirtschaftspolitischen Ideen und materiellen Interessen, hätten die verschiedenen Befürworter einer Kapitalstockförderung oft nicht unterschiedlicher sein können. Geschichten über die wirtschaftlichen Ursachen des früheren Niedergangs Großbritanniens allerdings fanden sich sowohl bei progressiven Bewunderern des japanischen Modells als auch bei tendenziell konservativen Ökonomen. In späteren Auseinandersetzungen um die Handelspolitik hingegen waren es genau diese Bedrohungsszenarien, mit der man die Regierung unter Zugzwang zu setzen versuchte. »Die Zukunft der amerikanischen Industrie " wurde in diesem Sinn einerseits zu einem politischen Trumpf neoliberaler Reformer und andererseits selbst zu einer Restriktion ihres politischen Handelns.

Wie verhält sich eine derartige pragmatische Perspektive auf Rechtfertigungsstrategien zu Forschungsansätzen, die die Rolle von Ideen in der Politik betonen? Die neuere Literatur zur Rolle von Ideen in der Wirtschaftspolitik basiert insbesondere

78 US Trade Representative 2018, S. 4.

79 Beckert 2016; Ergen 2015.

Leviathan, 47. Jg., 2/2019 
auf der Untersuchung von Policy-Paradigmen und Denksystemen. ${ }^{80}$ Theoretisch beschreibt diese Literatur unidirektionale und unmittelbare Beeinflussungsprozesse politischen Handelns durch Wissensstrukturen und Ideensysteme. Blickt man hingegen auf empirische Studien, finden sich dort allerlei Komplikationen - etwa halbherzig implementiertes ökonomisches Rezeptwissen, nationale Hybride aus unterschiedlichen Policy-Paradigmen und ständiges politisches Experimentieren. Die Untersuchung der Karrieren von Zukunftserzählungen kann ein möglicher Weg sein, einige solcher Komplikationen im Zusammenspiel zwischen Ideen und politischen Prozessen systematischer zu verstehen. Zukunftserzählungen verbinden unterschiedliche Interessen und fügen sich in verschiedene Diskurse. Dadurch können sie die Entstehung historisch besonderer Mischformen aus vermeintlich widersprüchlichen wirtschaftspolitischen Ansätzen bedingen.

\section{Literatur}

Beckert, Jens 2016. Imagined futures: fictional expectations and capitalist dynamics. Cambridge: Harvard University Press.

Beckert, Jens 2019. »The exhausted futures of neoliberalism. From promissory legitimacy to social anomy ", in Journal of Cultural Economy, Online First. DOI: 10.1080/17530350.2019.1574867.

Beramendi, Pablo; Häusermann, Silja; Kitschelt, Herbert; Kriesi, Hanspeter. Hrsg. 2015. The politics of advanced capitalism. Cambridge: Cambridge University Press.

Block, Fred 2009. "Read their lips: taxation and the right-wing agenda ", in The New Fiscal Sociology: taxation in comparative and historical perspective, hrsg. v. Martin, Isaac W.; Mehrotra, Ajay K.; Prasad, Monica, S. 68-85. Cambridge: Cambridge University Press.

Blyth, Mark 2002. Great transformations: economic ideas and institutional change in the twentieth century. Cambridge: Cambridge University Press.

Borrus, Michael 1983. »The politics of competitive erosion in the U.S. steel industry «, in American industry in international competition. Government policies and corporate strategies, hrsg. v. Zysman, John; Tyson, Laura, S. 60-105. Ithaca: Cornell University Press.

Campbell, John L.; Pedersen, Ove K. 2015. "Policy ideas, knowledge regimes and comparative political economy «, in Socio-Economic Review 13, 4, S. 679-701.

Carleson, Robert B. 1982. Memorandum: the administration plan for enterprise zones. Collection: BE003; Industry; 058000-066262: Ronald Reagan Presidential Library.

Carter, Jimmy 1979. Industrial innovation initiatives. Message to the Congress on Administration Actions and Proposals. 31. Oktober 1979. Washington.

Chorev, Nitsan 2007. Remaking U.S. trade policy: from protectionism to globalization. Ithaca: Cornell University Press.

Christophers, Brett 2016. The great leveler: capitalism and competition in the Court of Law. Cambridge: Harvard University Press.

Council of Economic Advisers 1983. Annual report of the Council of Economic Advisers. Economic report of the President. Washington.

Culpepper, Pepper D. 2010. Quiet politics and business power: corporate control in Europe and Japan. Cambridge: Cambridge University Press.

Dertouzos, Michael L.; Lester, Richard K.; Solow, Robert M. 1991. Made in America: regaining the productive edge. Cambridge: MIT Press.

Dobbin, Frank 1994. Forging industrial policy: The United States, Britain, and France in the railway age. Cambridge: Cambridge University Press.

Dobbin, Frank; Jung, Jiwook 2010. »The misapplication of Mr. Michael Jensen: how agency theory brought down the economy and why it might again «, in Markets on trial: the eco-

80 Dobbin 1994; Hall 1993; Campbell, Pedersen 2015. 
nomic sociology of the U.S. financial crisis. Research in the sociology of organizations $30 \mathrm{~b}$, hrsg. v. Lounsbury, Michael; Hirsch, Paul M., S. 29-64. Bingley: Emerald.

Eisner, Robert 1977. "Capital shortage: myth and reality «, in American Economic Review 67, 1, S. $110-115$.

Ergen, Timur 2015. Große Hoffnungen und brüchige Koalitionen. Industrie, Politik und die schwierige Durchsetzung der Photovoltaik. Frankfurt a. M., New York: Campus.

Ergen, Timur; Kohl, Sebastian 2019. "Varieties of economization in competition policy: institutional change in German and American antitrust, 1960-2000 ", in Review of International Political Economy, Online First. DOI:10.1080/09692290.2018.1563557.

Fisher, Peter S. 1985. »Corporate tax incentives: the American version of industrial policy «, in Journal of Economic Issues 19, 1, S. 1-19.

Fourcade, Marion; Babb, Sarah L. 2002: »The rebirth of the liberal creed: paths to neoliberalism in four countries ", in American Journal of Sociology 108, 3, S. 533-579.

Fraser, Nancy 2017. "From progressive neoliberalism to Trump - and beyond ", in American Affairs 1, 4, S. 46-64.

Friedman, Milton 1987. »Outdoing Smoot-Hawley«, in Wall Street Journal vom 20. April 1987.

Fullerton, Don; Walker, Charls E.; Long, Russell B. 1994. "Tax policy ", in American economic policy in the 1980 s, hrsg. v. Feldstein, Martin, S. 165-234. Chicago: University of Chicago Press.

Ganghof, Steffen 2000. »Adjusting national tax policy to economic internationalization: strategies and outcomes ", in From vulnerability to competitiveness. Welfare and work in the global economy, Band II, hrsg. v. Scharpf, Fritz W.; Schmidt, Vivian A., S. 597-564. Oxford: Oxford University Press.

Ganghof, Steffen 2004. Wer regiert in der Steuerpolitik? Einkommensteuerreform zwischen internationalem Wettbewerb und nationalen Verteilungskonflikten. Frankfurt a. M., New York: Campus.

Genschel, Philipp 2002. Stenerharmonisierung und Stenerwettbewerb in der Europäischen Union. Frankfurt a. M., New York: Campus.

Genschel, Philipp; Schwarz, Peter 2011. "Tax competition: a literature review ", in Socio-Economic Review 9, S. 339-370.

Genschel, Philipp; Seelkopf, Laura 2015. »The competition state: the modern state in a global economy", in The Oxford handbook of transformations of the state, hrsg. v. Leibfried, Stephan et al., S. 237-252. Oxford: Oxford University Press.

Gerring, John 2001. Social science methodology: a criterial framework. Cambridge: Cambridge University Press.

Gilpin, Robert 1981. War and change in world politics. Cambridge: Cambridge University Press.

Graham, Otis L. 1992. Losing time: the industrial policy debate. Cambridge: Harvard University Press.

Hacker, Jacob S.; Pierson, Paul 2010. Winner-take-all politics: how Washington made the rich richer - and turned its back on the middle class. New York: Simon \& Schuster.

Hacker, Jacob S.; Pierson, Paul; Thelen, Kathleen 2015. »Drift and conversion: hidden faces of institutional change", in Advances of comparative-historical analysis, hrsg. v. Mahoney, James; Thelen, Kathleen, S. 180-208. Cambridge: Cambridge University Press.

Hall, Peter A. 1993. »Policy paradigms, social learning, and the state: the case of economic policymaking in Britain ", in Comparative Politics 25, 3, S. 275-296.

Hoerr, John P. 1988. And the wolf finally came: the decline of the American steel industry. Pittsburgh: University of Pittsburgh Press.

Hutcheson, Rick 1977. An economic nightmare. Collection: Office of Staff Secretary; Series: Presidential Files; Folder: 12/5/77 [2]; Container 53: Jimmy Carter Presidential Library.

Katzenstein, Peter J. 1985. Small states in world markets. Industrial policy in Europe. Ithaca, London: Cornell University Press.

Kennedy, Paul 1988. The rise and fall of great powers: economic change and military conflict from 1500 to 2000. London: Unwin Hyman.

Kindleberger, Charles P. 1973. »An American economic climacteric? «, in Challenge 16, 6, S. 3544. 
Krippner, Greta 2012. Capitalizing on crisis: the political origins of the rise of finance. Cambridge: Harvard University Press.

Krugman, Paul 1994. »Competitiveness: a dangerous obsession «, in Foreign Affairs 73, 2, S. 28 44.

Lindert, Peter H. 2004. Growing public: social spending and economic growth since the eighteenth century. Band 1: The story. Cambridge: Cambridge University Press.

Martin, Cathie Jo 1990. Shifting the burden: the struggle over growth and corporate taxation. Chicago: Chicago University Press.

Martin, Isaac W. 2008. The permanent tax revolt: how the property tax transformed American politics. Stanford: Stanford University Press.

Martin, Isaac W.; Mehrotra, Ajay K.; Prasad, Monica. Hrsg. 2009. The New Fiscal Sociology: taxation in comparative and historical perspective. Cambridge: Cambridge University Press.

McIntyre, Robert S.; Folen, Robert 1984. Corporate income taxes in the Reagan years. A study of three years of legalized tax avoidance. Washington: Citizens for Tax Justice.

McQuarrie, Michael 2017. »The revolt of the Rust Belt: place and politics in the age of anger ", in British Journal of Sociology 68, S1, S. 120-152.

Minarik, Joseph J. 1990. Making America's budget policy: from the 1980 s to the 1990 s. Armonk: Sharpe.

Nixon, Richard M. 1971. Statement about the Revenue Act of 1971. 10. Dezember 1971. Washington.

Patashnik, Eric M. 2008. Reforms at risk: what happens after major policy changes are enacted. Princeton: Princeton University Press.

Patashnik, Eric M.; Zelizer, Julian E. 2013. »The struggle to remake politics: liberal reform and the limits of policy feedback in the contemporary American state «, in Perspectives on Politics 11, 4, S. 1071-1087.

Petrocik, John R. 1996. »Issue ownership in presidential elections, with a 1980 case study «, in American Journal of Political Science 40, 3, S. 825-850.

Piketty, Thomas 2014. Capital in the twenty-first century. Cambridge: Harvard University Press.

Piketty, Thomas; Saez, Emmanuel 2007: "How progressive is the U.S. federal tax system? A historical and international perspective ", in Journal of Economic Perspectives 21, 1, S. 3-24.

Polanyi, Karl 2001 [1944]. The great transformation: the political and economic origins of our time. Boston: Beacon Press.

Popp Berman, Elizabeth; Pagnucco, Nicholas 2010. "Economic ideas and the political process: debating tax cuts in the U.S. House of Representatives, 1962-1981 «, in Politics \& Society 38, 3, S. 347-372.

Prasad, Monica 2006. The politics of free markets: the rise of neoliberal economic policies in Britain, France, Germany, and the United States. Chicago: University of Chicago Press.

Prasad, Monica 2018. Starving the beast: Ronald Reagan and the tax cut revolution. New York: Sage Foundation Press.

Rademacher, Inga 2017. Common ground: justifications of neoliberal tax cuts in the US and Germany. Inauguraldissertation, Universität Osnabrück.

Reagan, Ronald 1981. Inaugural address. 20. Januar 1981. Washington.

Reagan, Ronald 1983. Statement on establishment of the President's commission on industrial competitiveness. 4. August 1983. Washington.

Reagan, Ronald 1985. Presidential address: President's export council. 23. September 1985. Draft: 9/20/85. Collection: Stucky, Edward: Files; Box 12; Trade Speech, 9/19/1985 (2 of 3): Ronald Reagan Presidential Library.

Richardson, J. David; Olmer, Lionel; Stern, Paula 1994. "Trade policy «, in American economic policy in the 1980 s, hrsg. v. Feldstein, Martin, S. 627-690. Chicago: University of Chicago Press.

Rodgers, Daniel T. 2011. Age of fracture. Cambridge: Harvard University Press.

Rodgers, Daniel T. 2018. » The uses and abuses of 'neoliberalism«", in Dissent Magazine, Winter 2018.

Schäfer, Armin 2008. Krisentheorien der Demokratie: Unregierbarkeit, Spätkapitalismus und Postdemokratie. Köln: Max-Planck-Institut für Gesellschaftsforschung. 
Schmidt, Vivian A. 2008. "Discursive institutionalism: the explanatory power of ideas and discourse ", in Annual Review of Political Science 11, S. 303-326.

Simmons, Beth; Dobbin, Frank; Garrett, Geoffrey 2007. »Introduction: the diffusion of liberalization ", in The global diffusion of markets and democracy, hrsg. v. Simmons, Beth; Dobbin, Frank; Garrett, Geoffrey, S. 1-63. Cambridge: Cambridge University Press.

Steinmo, Sven 1989. "Political institutions and tax policy in the United States, Sweden, and Britain ", in World Politics 41, 4, S. 500-535.

Streeck, Wolfgang 2011. »The crises of democratic capitalism «, in New Left Review 71, S. 5-29.

Streeck, Wolfgang; Elsässer, Lea 2016. »Monetary disunion: the domestic politics of Euroland «, in Journal of European Public Policy 23, 1, S. 1-24.

Swank, Duane 2006. " Tax policy in an era of internationalization: explaining the spread of neoliberalism ", in International Organization 60, 4, S. 847-882.

Swank, Duane; Steinmo, Sven 2002. »The new political economy of taxation in advanced capitalist democracies ", in American Journal of Political Science 46, 3, S. 642-655.

Tempalski, Jerry 2006. Revenue effects of major tax bills. Washington: Department of the Treasury, Office of Tax Analysis.

Tempalski, Jerry 2013. Revenue effects of major tax bills. Updated tables for all 2012 bills. Washington: Department of the Treasury, Office of Tax Analysis.

The White House 1981. America's new beginning: a program for economic recovery. 18 . Februar 1981. Washington.

The White House 1985. Press release draft: trade strike force. Collection: Stucky, Edward: Files; Box 12; Trade Strike Force (1 of 2): Ronald Reagan Presidential Library.

Thurow, Lester C. 2001 [1980]. The zero-sum society. Distribution and the possibilities for economic change. New York: Basic Books.

US Congress 1977. The role of federal tax policy in stimulating capital formation and economic growth. Hearings before the Subcommittee on Economic Growth and Stabilization of the Joint Economic Committee. First session. Ninety-fifth congress. Washington.

US Congress 1981. Tax aspects of the President's economic program. Hearings before the Committee on Ways and Means. US House of Representatives. Three sessions. Ninety-seventh congress. Washington.

US Trade Representative 2018. 2017 report to Congress on China's WTO compliance. Washington.

Whitehead, Alfred N. 1967 [1926]. Science and the modern world. New York: The Free Press. 
Zusammenfassung: Der Beitrag führt vor, wie Geschichten über die kollektive wirtschaftliche Zukunft zur Mobilisierung politischer Unterstützung für neoliberale Reformen genutzt wurden. Am Beispiel des Economic Recovery Tax Acts von 1981 wird gezeigt, wie die Regierung Ronald Reagans industrielle Niedergangsszenarien aufgriff, um Steuersenkungen durchzusetzen. Diese Rechtfertigungsstrategie rächte sich in der Folge, als andauernde industrielle Probleme in zunehmenden politischen Druck zur Einrichtung von Handelsbeschränkungen mündeten. Der Beitrag ist relevant für das breitere Verständnis der Durchsetzung umstrittener Reformen, indem er zeigt, dass Erzählungen über die Zukunft gesellschaftliche Koalitionen verschieben können.

Stichworte: Neoliberalismus, Ronald Reagan, Steuerpolitik, Erwartungen, Handelspolitik, Industriepolitik, Diskurse

\section{Visions of economic decline and neoliberal reform}

Summary: The article shows how stories about collective economic futures have been used to mobilize support for neoliberal reforms. An analysis of the Economic Recovery Tax Act of 1981 demonstrates that the Reagan administration invoked stories about economic decline to enact business tax cuts. This strategy backfired as continued industrial problems led to increasing demands for interventionist trade policies. The article contributes to the broader understanding of the passage of contentious reforms by demonstrating that stories about the future can alter social coalitions.

Keywords: neoliberalism, Ronald Reagan, tax policy, expectations, trade policy, industrial policy, discourse

Autor

Timur Ergen

Max-Planck-Institut für Gesellschaftsforschung (MPIfG)

Paulstraße 3

50676 Köln

te@mpifg.de 